\title{
Sistemas farmacêuticos gastrorretentivos flutuantes
}

\author{
Pedro Miguel da Costa Barrocas ${ }^{1}$, Delfim Fernando Gonçalves Santos ${ }^{1}$, Domingos Carvalho \\ Ferreira1, Pedro Miguel Barata de Silva Coelho ${ }^{1,2}$, Rita Cristina Sanches Oliveira ${ }^{1,2}$, \\ Francisco José de Baptista Veiga*,3
}

${ }^{1}$ Faculdade de Farmácia da Universidade do Porto, Porto, Portugal, 2Faculdade de Ciências da Saúde da Universidade Fernando Pessoa, Porto, Portugal, ${ }^{3}$ Faculdade de Farmácia da Universidade de Coimbra, Coimbra, Portugal

*Correspondência:

F. Veiga

Faculdade de Farmácia

Universidade de Coimbra

3000-295 - Coimbra, Portugal

E-mail: fveiga@ci.uc.pt
Freqüentemente recorre-se à produção de sistemas gastrorretentivos para modular a liberação de fármacos a partir de sistemas farmacêuticos com vistas ao aumento do tempo de permanência do fármaco no trato gastrointestinal. Umas das estratégias mais interessantes passa pela produção de sistemas flutuantes. Estes podem ser classificados em dois grupos: sistemas flutuantes efervescentes e sistemas flutuantes não-efervecentes. Neste artigo apresenta-se uma revisão bibliográfica do que tem sido produzido nesta área nos últimos anos.
Uniterm0s

- Sistemas gastorretentivos

- Sistemas flutuantes

- Sistemas efervescentes

- Formas farmacêuticas/ liberação modificada

\section{INTRODUÇÃO}

O desenvolvimento de novos sistemas terapêuticos tem como objetivo o aumento da biodisponibilidade dos fármacos, com o conseqüente aumento do efeito farmacológico e diminuição dos efeitos secundários. No entanto, estes objetivos são dificultados por impedimentos fisiológicos tais como a dificuldade em manter a forma farmacêutica no local ótimo para a sua absorção. Estima-se que cerca de um terço dos fármacos atualmente administrados oralmente apresenta baixa biodisponibilidade quando administrado no cólon.

O prolongamento do tempo de retenção gástrica (GRT) em determinadas circunstâncias pode ser útil para aumentar o efeito terapêutico de um fármaco. No caso de fármacos absorvidos sobretudo no estômago ou na parte proximal do intestino delgado ou ainda no caso de fármacos que sejam degradados em $\mathrm{pH}$ alcalino as vantagens deste tipo de dispositivos tornam-se evidentes (Rouge, Buri, Doelker, 1996; Fell, Whitehead, Collet, 2000; Matharu, Sanghavi, 1992). Outras vantagens são o aumento da biodisponibilidade, da eficácia terapêutica e redução da dosagem (Fell, 1999; Baumgartner et al., 2000). Por exemplo, no tratamento de infecções por Helicobacter pylori as vantagens deste tipo de dispositivos tornam-se evidentes, visto que permitem uma liberação dos princípios ativos no local de atuação por um período de tempo maior (Yang, Eshragi, Fassihi, 1999).

No entanto os sistemas gastrorretentivos possuem algumas desvantagens: não podem ser utilizados no caso de fármacos que possam provocar lesões gástricas (AINE's) ou que se degradem em ambiente ácido. Por outro lado, este tipo de sistema não apresenta vantagens em relação aos sistemas de liberação convencional no caso de fármacos que sejam bem absorvidos ao longo de todo o trato gastrointestinal

\section{SISTEMAS GASTRORRETENTIVOS}

Os sistemas gastrorretentivos apresentam várias formas de alcançar o mesmo objetivo: o aumento do tempo de permanência em nível gástrico. Assim, existem vários tipos 
de dispositivos como mucoadesivos, intumescíveis, altadensidade e flutuantes, todos com diferentes formas de atuação.

Os sistemas mucoadesivos aumentam o GRT, pois aderem à membrana da mucosa gástrica. A bioadesão em tecidos moles, utilizando polímeros naturais ou sintéticos, é explorada para controlar e prolongar o GRT e a liberação de fármacos (Robinson et al., 1985). A adesão dos polímeros à membrana mucosa pode ser mediada por processos de hidratação, ligação química ou mecânica, ou mediada por receptor (Robinson, Park, 1984).

Os sistemas intumescíveis possuem polímeros que intumescem quando em contacto com o fluido gástrico aumentando de tamanho, de modo que não consigam passar através do piloro. Assim, o sistema fica retido no estômago por um longo período de tempo. O equilíbrio entre o intumescimento e a velocidade de degradação do polímero é essencial para obter boa resposta farmacológica e evitar efeitos secundários.

Os sistemas de alta-densidade têm como objetivo alojar-se no ambiente do estômago e resistir aos movimentos peristálticos. Estes sistemas possuem uma densidade superior ou igual a $1,3 \mathrm{~g} / \mathrm{mL}$, que é superior à densidade do fluido gástrico (Bechgaard, Baggesen, 1980).

Os sistemas flutuantes devem flutuar no conteúdo gástrico, aumentando, assim, o GRT. Estes sistemas são freqüentemente classificados em efervescentes e não-efervescentes.

\section{Sistemas farmacêuticos flutuantes efervescentes}

Os dispositivos flutuantes são constituídos por matriz composta por polímeros expansíveis como o Methocel ${ }^{\circledR}$ ou polissacarídeos, misturados com compostos geradores de gás, como o bicarbonato de sódio e ácido cítrico ou tartárico, (Rubinstein, Friend, 1994), ou por matriz que contem uma camada de líquido que gasifica à temperatura corpórea (Ritsehel,1991; Michaels, 1974). O componente que dá origem ao anidrido carbônico pode estar intimamente misturado no interior da matriz do comprimido, dando origem a matriz monocamada (Sheth, Tossounian, 1979). Existem, ainda, comprimidos de dupla camada que contêm a substância geradora de gás numa camada, hidrocolóide e o fármaco em outra camada, formulado de modo a ocorrer um efeito de liberação modificada. As matrizes são preparadas de modo a que, quando chegam ao estômago, o anidrido carbônico é liberado devido à acidez do conteúdo gástrico e incorporado no hidrocolóide gelificado. Isto causa a queda da densidade específica, o que permite um movimento em direção ao topo do líquido e causa a flutuação (Rubinstein, Friend, 1994). Os dispo- sitivos dotados de um mecanismo de geração de gás interno deveriam ter melhor flutuação e melhores características de liberação de fármaco. Todavia, a otimização das características de liberação pode influenciar a flutuação e, portanto, pode ser necessário separar o controle da flutuação daquele da cinética de liberação do fármaco durante o tempo de otimização da formulação (Rouge, Burl, Doelker,1996).

Umezawa, em 1978, desenvolveu microcápsulas flutuantes. A liberação de dióxido de carbono, quando ocorre o contato do dispositivo com o ambiente ácido do estômago, permitia que a microcápsula flutuasse. O núcleo das microcápsulas era constituído por um comprimido de bicarbonato de sódio misturado com lactose e polivinilpirrolidona, revestido por repetidas aplicações por spray de uma solução de HPMC a $2 \%$ em metanol. O núcleo era revestido externamente com pepsatina, que permitia um aumento do tempo de permanência no estômago. A conclusão foi que a administração de um dispositivo que continha 50-200 mg de pepsatina por dose permitia a liberação de uma quantidade suficiente de pepsatina que suprimia a atividade pepsínica em pacientes tratados de úlcera gástrica ou duodenal.

Todd e Fryers, em 1979, descreveram uma formulação contendo colestiramina anidra (uma resina trocadora aniônica), ácido algínico/alginato de sódio de baixo grau de viscosidade, ácido cítrico e quantidade suficiente de carbonato de sódio ou bicarbonato de modo a neutralizar os grupos ácidos do ácido algínico e cítrico. A resina era carregada com o bicarbonato ou carbonato e com ácidos e revestida com os componentes restantes. Após administração por via oral o contato com a saliva e, mais tarde, com o suco gástrico, provoca a reação do bicarbonato com os ácidos levando à formação de dióxido de carbono, que fica aprisionado na camada de ácido algínico/alginato de sódio hidratada.

Stockwell, Davis e Walkker, em 1986, desenvolveram cápsulas flutuantes contendo mistura de alginato e de bicarbonato de sódio. O sistema flutuava durante os testes in vitro como conseqüência da geração de dióxido de carbono incorporado na estrutura do gel hidratante, após a exposição a um ambiente ácido.

Inouye, Machida e Sannan, em 1989, utilizaram dois tipos de quitosana com diversos graus de diacetilação (quitosanas $\mathrm{H}$ e L) e predenisolona como fármaco de referência. Embora ambos os tipos de quitosana garantissem a liberação controlada do fármaco em meio de dissolução ácido e permitissem obter uma rápida flutuação do dispositivo, a liberação a partir da formulação que continha quitosana $\mathrm{L}$ era mais lenta da que com a formulação à base de quitosana H. Num estudo follow-up conduzido pelo 
mesmo grupo, as propriedades de liberação eram controladas regulando o conteúdo de quitosana dos comprimidos ou a espessura da membrana de quitosana $\mathrm{L}$ dos preparados laminados. Foram, ainda, testados ainda comprimidos de liberação controlada de indometacina por Miyazaki et al., em 1988.

Ichinawa et al., em 1989, descreveram uma cápsula contendo uma série de grânulos com tempo de permanência variável no estômago. Os grânulos eram constituídos por um núcleo contendo o fármaco revestido por dupla camada. A camada interna era efervescente e a externa constituída por um filme expansível contendo um polímero que permitia a passagem do suco gástrico e expandia como conseqüência da reação do suco gástrico com a camada efervescente. Por outro lado, a camada efervescente era dividida em outras duas sub-camadas, uma interna contendo bicarbonato e uma externa contendo um acido orgânico. A liberação de dióxido de carbono servia para acelerar a hidratação dos comprimidos flutuantes, essencial para a formação de um hidrogel bioadesivo. Isto fornece um mecanismo auxiliar (bioadesão) de retenção do dispositivo no estômago, para além da flutuação.

Atyabi et al., $(1994,1996)$ desenvolveram um sistema flutuante utilizando uma resina de troca de íons. O sistema consistia na utilização de grânulos da resina carregada com bicarbonato e com um fármaco de carga negativa, que se ligava à resina. As microesferas resultantes eram depois encapsuladas numa membrana semipermeável com o objetivo de evitar a perda rápida de dióxido de carbono. No momento do contato com o meio ácido do estômago verificava-se uma troca de íons cloro e bicarbonato. Como conseqüência desta reação o dióxido de carbono liberado era incorporado na membrana, transportando as microesferas para a parte superior do conteúdo gástrico e dando origem a uma camada flutuante de grânulos de resina. Os grânulos não revestidos, pelo contrário, imergiam imediatamente. A análise do GRT mediante cintilografia demonstrou que o tempo de retenção no estômago era prolongado de maneira substancial em relação ao controle, quando o dispositivo era administrado após uma refeição ligeira e preferencialmente líquida. Por outro lado, o sistema era capaz de liberar lentamente o fármaco, propriedade que ampliava o potencial flutuante para fármacos com carga negativa, dado que podem facilmente se ligar à resina em combinação com o bicarbonato.

Yang, Esharagi e Fassihi, em 1999, desenvolveram um novo tipo de comprimidos de liberação modificada, contendo três camadas assimétricas, para o tratamento da infecção de Helicobacter pylori contendo três fármacos (tetraciclina, metronidazol e um sal de bismuto). Uma das camadas tem característica efervescente conferindo capa- cidade de flutuar aos comprimidos, conseguindo, assim, prolongar o seu GRT. Esta camada é constituída por mistura de óxido de polietileno, bicarbonato de sódio e carbonato de cálcio, tendo a capacidade de quando em contato com o meio ácido gerar gás que fica aprisonado. A camada interna é constituída por hidroxipropilcelulose e o óxido de polietileno, que são os polímeros responsáveis por regular a liberação de fármaco, nos quais a tetraciclina e o metronidazol foram incorporados de modo a que a sua liberação seja controlada, enquanto que os sais de bismuto foram incorporados na camada externa de modo a serem imediatamente liberados. Os resultados demonstraram que a tetraciclina e o metronidazol foram liberados de forma controlada por um período de 6 a 8 horas, mantendo os comprimidos flutuando. A capacidade de flutuação aumentou o tempo de permanência gástrica e ajudou a manter a concentração da tetraciclina e do metronidazol no local de ação. O sistema desenvolvido tem potencial para aumentar a eficácia da terapia e aumentar a aceitabilidade do tratamento pelos pacientes.

Wei, Yu e Bi, em 2001 desenvolveram pastilhas flutuantes de cisaprida com duas camadas. Uma das camadas é constituída por bicarbonato de sódio, HMPC e amido, que se expande rapidamente quando em contato com fluido gástrico artificial. Por outro lado, a reação do meio ácido com o bicarbonato gera dióxido de carbono, que fica aprisionado pelo $\mathrm{HMPC}$, conferindo às pastilhas capacidade de flutuar. A outra camada contém o fármaco. A liberação de fármaco in vitro e a capacidade de flutuação foram determinadas. A liberação in vitro de fármaco era controlada pela quantidade de HMPC adicionada à camada contendo o fármaco. Geralmente, quanto maior a proporção de HMPC mais lenta é a liberação do fármaco. Demonstrouse que estas pastilhas possuíam maior tempo de permanência ao nível gástrico e melhor perfil de liberação de fármaco do que os comprimidos de liberação retardada convencionais. A presença de duas camadas permitia regular independentemente a capacidade de flutuação e a capacidade de liberação de fármaco.

Umamahreshwari, Jain e Jain, em 2003, desenvolveram microcápsulas de colestiramina, revestidas com acetobutirato de celulose (CAB), com capacidade de flutuar devido à geração de dióxido de carbono quando expostas ao fluido gástrico. As microcápsulas também possuíam propriedades mucoadesivas. As microcápsulas foram preparadas carregando partículas de resinas trocadoras de íons com bicarbonato e ácido acetoidroxâmico seqüencialmente, que foram depois revestidas com $\mathrm{CAB}$ através de um processo de evaporação do solvente. A concentração de fármaco foi monitorada de modo a manter a capacidade de flutuação e a concentração mínima efetiva. A influência da 
razão CAB:fármaco-resina $(2: 1,4: 1,6: 1 \mathrm{w} / \mathrm{w})$ no tamanho de partícula, tempo de flutuação e nas características de liberação do fármaco também foram determinadas. Inicialmente o meio de dissolução penetra no revestimento da microcápsula atingindo a resina, o revestimento expandese e diminui a sua densidade. A interação entre o fluido ácido e o bicarbonato leva à geração de dióxido de carbono, que fica aprisionado na camada de revestimento. O tempo de flutuação das microcápsulas revestidas com CAB revelou-se maior do que o das microcápsulas não-revestidas e maiores tempos de flutuação foram observados na maior razão polímero:complexo fármaco-resina (6:1). Com o aumento da espessura do revestimento verificou-se um aumento no tamanho de partícula, mas uma diminuição na capacidade de liberação de fármaco. A liberação de fármaco era maior em fluido gástrico artificial do que em fluido intestinal artificial. As microcápsulas distribuíram-se no estômago e exibiram elevados períodos de residência gástrica devido à capacidade de mucoadesão. Estes resultados indicam que as microcápsulas revestidas por CAB têm capacidade de flutuação e mucoadesão e, portanto, apresentam-se promissoras no tratamento de infecções por Helicobacter pylori.

Dave, Amin e Patel, em 2004, descreveram um sistema de liberação sustentada de ranitidina em que a flutuação é conseguida pelo efeito combinado do bicarbonato de sódio (agente gerador de gás), ácido cítrico e ácido esteárico com polímeros hidrofílicos como o HPMC $\mathrm{K} 4 \mathrm{M}$ e a goma xantana. O gás formado é aprisionado na camada gelificada permitindo que o comprimido flutue por mais de oito horas, após um período de latência de cerca de 2 minutos, necessário para a formação de gás e hidratação do polímero.

Técnica semelhante foi utilizada por Xiaoqiang, Minjie, Feng e Yiqiao, (2006), recorrendo aos carbômeros e ao HPMC como polímeros hidrofílicos e ao bicarbonato de sódio como gerador de gás, com o objetivo de preparar comprimidos efervescentes para a liberação modificada de cloreto de fenoporlamina.

\section{Sistemas farmacêuticos flutuantes não-efervescentes}

Os excipientes mais utilizados nos sistemas flutuantes não-efervescentes são hidrocolóides gelificantes, a celulose fortemente expansível, os polissacarídeos e polímeros formadores de matrizes como o poliacrilato, o polimetacrilato, o policarbonato e o poliestireno. Sheth e Tossounian, em 1984, lançaram a hipótese de que quando este tipo de dispositivos entra em contato com um meio aquoso, o hidrocolóide sofre um processo de hidratação formando inicialmente um gel na superfície do dispositivo. À medida que ocorre a hidratação o extrato hidrocolóide dá origem a um estrato de gelificado. Em conseqüência da hidratação, o fármaco dissolve-se internamente e difundese para o exterior, sendo a sua difusão controlada pela camada de hidrocolóide gelificado. O estrato gelificado expande-se em contato com o fluido gástrico após a sua administração por via oral e a sua forma mantém uma integridade relativa e uma densidade aparente inferior à unidade. $\mathrm{O}$ ar incorporado no polímero expandido confere capacidade de flutuação a este dispositivo. Um dos métodos de formular estes dispositivos baseia-se na mistura do fármaco com o hidrocolóide gelificante.

Numa patente concedida à empresa japonesa Eisai Co. Ltd, desenvolvida por Watanabe et al., em 1976, foi descrito um invólucro revestido, flutuante, constituído essencialmente por um revestimento globular, oco, de poliestireno. $\mathrm{O}$ dispositivo obtido era flutuante, devido à uma camada vazia da cápsula, e conseguia alcançar tempos de trânsito prolongado no estômago. A superfície externa do revestimento era revestida com duas camadas, a primeira era constituída por acetoftalato de celulose e a segunda por etilcelulose e HMPC adicionada de uma quantidade eficaz de um princípio ativo selecionado entre os seguintes grupos: um inibidor da secreção gástrica; uma substância neutralizante da acidez gástrica; um inibidor antipepsina. No entanto, tornava-se muito difícil incorporar o fármaco no interior deste sistema.

Harrigan, em 1977, descreveu um sistema flutuante, constituído por um reservatório para o fármaco encapsulado e uma câmara de flutuação. O reservatório do fármaco era um compartimento microporoso com distribuição de poros nas superfícies superior e inferior. As paredes periféricas do compartimento usado como reservatório do fármaco eram perfeitamente seladas de modo a evitar qualquer contato físico do fármaco não dissolvido com as paredes do estômago. A presença de uma câmara de flutuação anexa ao reservatório do fármaco torna possível a flutuação do sistema no fluido gástrico.

Sheth e Tossounian, em 1978, desenvolveram uma cápsula hidrodinamicamente balanceada (HBS) contendo mistura de fármaco e hidrocolóide. Em contato com o fluido gástrico, a parte externa da cápsula dissolvia-se e a mistura de fármaco e hidrocolóide formavam uma barreira gelificada, que lhe permitia a manutenção ao nível gástrico por tempo prolongado. Os mesmos autores desenvolveram comprimidos flutuantes de liberação prolongada, que se mantinham hidrodinamicamente balançeados no interior do estômago por tempo prolongado, até liberação completa da dose contida. Os comprimidos continham um princípio ativo, $0-80 \%$ de material inerte, e $20-75 \%$ de peso era composto por um hidrocolóide como a metilcelulose, 
HPC, HPMC, hidroxietilcelulose e carboximetilcelulose sódica, que, em contato com o fluido gástrico, formavam uma barreira de gel coloidal permeável à água na superfície dos comprimidos.

Mitra, em 1984, descreveu um dispositivo multi-camada, flexível e laminar, que flutuava no suco gástrico e possuía características de liberação modificada. O dispositivo consistia em pelo menos uma camada de base seca e independente composta por matriz polimérica hidroinsóluvel na qual se dispersava ou dissolvia o fármaco, e uma camada barreira aplicada sobre a camada base. A camada barreira era constituída por um polímero ou um co-polímero hidrossóluvel permeável ao fármaco e à água. Ambas as camadas eram seladas na sua periferia de maneira a incorporar uma série de pequenos sacos de ar, que tornavam possível a flutuação do filme laminado. Era possível modular o tempo de flutuação desejado e a velocidade de liberação mediante a adequada seleção da matriz polimérica. Os polímeros como o policarbonato eram utilizados para modular o grau de flutuação no fluido gástrico, de modo a liberar o conteúdo de fármaco no interior da forma farmacêutica por um período de tempo prolongado.

Ushimaru, Nakamiehi e Saito, em 1987, desenvolveram uma cápsula de liberação controlada, na qual utilizaram mistura do fármaco com um derivado da celulose, do amido ou do ácido acrílico, que gelificasse na água, e um glicerídeo de ácidos graxos superiores e seus ésteres, um álcool superior e seus ésteres, ou mistura de vários destes produtos que permanecesse sólida à temperatura ambiente. A cápsula era enchida com esta mistura e depois aquecida até temperatura superior ao ponto de fusão do componente graxo e, posteriormente, sujeita a um arrefecimento e conseqüente solidificação da mistura.

Desai e Bolton $(1989,1993)$ desenvolveram comprimidos de teofilina de liberação modificada utilizando ágar e óleo mineral leve. A quantidade de ágar necessário para formar os comprimidos era reduzida ( $2 \%$ por comprimido). O óleo mineral leve era essencial para garantir a liberação modificada dos comprimidos, devido à quantidade relativamente elevada de fármaco que era utilizada (75\%). Por outro lado, o óleo mineral leve presente na formulação servia para evitar que o ar incorporado na matriz gelatinosa fosse liberado quando em contato com o fluido gástrico, devido à sua hidrofobicidade.

Os comprimidos eram produzidos introduzindo-se mistura de fármaco/óleo mineral numa solução aquecida de gel ágar e vertendo a mistura resultante nos moldes para os comprimidos, que, após arrefecimento e secagem ao ar, davam origem aos comprimidos de liberação modificada. $\mathrm{O}$ ar incorporado na estrutura da matriz gelatinosa contribuía para reduzir a densidade e aumentar a liberação modificada dos comprimidos. O estudo indicou um outro aspecto importante da estrutura do gel ágar, que é o fato deste permitir obter comprimidos sem a necessidade de compressão, visto que o ágar confere dureza e friabilidade desejáveis e ao mesmo tempo controla as características de liberação do fármaco. Em outro estudo, os mesmos autores descreveram uma formulação análoga sem o emprego de óleo.

Kawashima et al. (1991, 1992), realizaram microesferas vazias com o fármaco carregado na camada externa, mediante um sistema de emulsificação-evaporação do solvente. Uma solução etanol/diclorometano de um fármaco juntamente com um polímero acrílico foi misturada com uma solução aquosa de álcool polivinílico (PVA) a $40^{\circ} \mathrm{C}$. A segunda solução era adicionada mediante agitação contínua com a primeira solução de modo a se obter uma emulsão com incorporação de ar. A fase gasosa gerada no interior do polímero depois da evaporação do diclorometano formava uma cavidade no interior da microesfera. Durante o ensaio in vitro, as microesferas vazias continuavam a flutuar na superfície do meio de dissolução aquoso ou ácido contendo um tensioativo por um período de mais de 12 horas. Além disso, o perfil de liberação do fármaco das microesferas vazias comportava-se como um sistema gastrorresistente e a velocidade de liberação do fármaco era controlada pela razão polímero/fármaco no interior do sistema.

Chitnis, Malshe e Lalla (1991) sintetizaram uma série de polímeros bioadesivos como polímeros reticulados de ácido metacrílico (PMA) e ácido acrílico (PAA). Os comprimidos flutuantes de mononitrato de isossorbida eram preparados por revestimento por imersão numa dispersão de Carbopol ${ }^{\circledR}$ ou uma dispersão de $0,5 \%$ de um polímero bioadesivo num gel de carbopol ${ }^{\circledR}$ a $0,5 \%$ e, posteriormente, colocados para secar ao ar. Os resultados indicaram que os comprimidos revestidos com o polímero bioadesivo possuíam melhores propriedades de adesão a um $\mathrm{pH}$ de 1,0 em relação aos comprimidos revestidos com a suspensão de Carbopol $^{\circledR}$. Além disso, os comprimidos revestidos possuíam uma densidade menor e, conseqüentemente, o revestimento polimérico conferia melhores características de flutuação a estes comprimidos.

Dennis, Timmins e Lee, em 1992, descreveram uma formulação em pó flutuante de liberação modificada. A formulação consistia num fármaco de carácter básico, um polímero dependente do $\mathrm{pH}$, neste caso um sal hidrossolúvel do ácido algínico (como o alginato de sódio ou de potássio), uma substância gelificante hidrocolóide independente do $\mathrm{pH}$ (como HMPC, metilcelulose, hidroxipropilcelulose ou mistura de mais substâncias) e um ligando. A formulação liberava o fármaco a uma velocidade controlada independentemente do $\mathrm{pH}$ do ambiente, sem produção de 
íon cálcio ou dióxido de carbono, e com um perfil de liberação semelhante a um comprimido de análoga composição. Esta formulação podia ser utilizada para encher cápsulas ou fazer comprimidos.

Franz e Oth (1993) descreveram um dispositivo de liberação modificada que consiste numa cápsula contendo uma formulação de dupla camada não comprimida. Uma das camadas era utilizada para a liberação do fármaco (misoprostrol), e a outra era utilizada como camada flutuante. Esta camada continha uma substância hidrocolóide gelificante como a hidroxipropilmetilcelulose (HMPC), que, em contato com o fluido gástrico, formava massa gelatinosa, que promovia a fusão da camada do fármaco e da camada flutuante de modo a promover a liberação modificada do fármaco. Verificou-se que este dispositivo possuía um tempo de permanência no fluido gástrico de 13 horas, e que liberava uma quantidade substancial de fármaco no estômago.

Thanoo, Sunny e Jayakrishnan, em 1993, desenvolveram microesferas de policarbonato contendo fármaco utilizando uma técnica baseada na evaporação do solvente. Mediante este procedimento foi possível alcançar uma dosagem de fármaco superior a $50 \%$. Por outro lado, aumentando a relação fármaco/polímero no interior da microsfera, aumentava-se a dimensão média das partículas e a velocidade de liberação do fármaco.

Whitehead, Fell e Collet, em 1996, desenvolveram um dispositivo utilizando alginato de cálcio liofilizado. As microesferas eram produzidas adicionando uma solução de alginato de sódio a uma solução cloreto de cálcio aquoso. No fim do processo de gelificação, as microesferas eram separadas da solução e congeladas imediatamente em nitrogênio líquido antes de serem liofilizadas a $-40^{\circ} \mathrm{C}$ por 24 horas. A medição do peso resultante indicava que as microesferas mantinham uma força de flutuação pelo menos durante 12 horas. Num estudo feito posteriormente, a capacidade de gastrorretenção das microesferas flutuantes foi analisada em sujeitos do sexo masculino após uma refeição, utilizando a técnica de gama-cintilografia, e confrontada com aquela dos grânulos não-flutuantes obtidos com o mesmo material. Em todos os indivíduos que tomaram a formulação flutuante o GRT era prolongado por mais de 5,5 horas, enquanto que as microesferas não-flutuantes apresentavam um GRT mais curto, com um tempo médio de 1 hora.

Iannucelli et al. (1998) descreveram um dispositivo múltiplo que continha um compartimento com ar. O dispositivo era composto por um núcleo de alginato de cálcio separado com uma camada intermédia de ar de uma membrana de alginato de cálcio ou alginato de cálcio/PVA. A estrutura porosa gerada pela dissolução do PVA, utilizado como aditivo hidrossóluvel na composição do revestimento, levava a um aumento da permeabilidade da membrana, evitando o colapso da camada intermediária de ar. Os resultados in vitro indicaram que a capacidade de flutuação aumentava com o incremento da concentração de PVAe da massa molecular.

Num estudo realizado por Soppimath et al., em 2001, desenvolveram-se microsferas ocas de acetato de celulose carregadas com quatro fármacos cardiovasculares (nifedipino, cloridrato de nicardipino, cloridrato de verapamil e dipiridamol) através de um processo de difusão-evaporação do solvente, utilizando uma emulsão O/A de acetato de etilo numa solução aquosa de álcool polivinílico a $0,05 \%$. As microesferas mostraram superfícies lisas, estrutura oca e a capacidade de flutuar por mais de 12 horas. Os fármacos carregados nas microesferas estavam no seu estado amorfo. As microesferas proporcionaram a liberação controlada de fármacos por mais de 12 horas, o que lhes permitiu a liberação de nifedipino e nicardipino por um período superior a 15 horas para controle efetivo da hipertensão.

Joseph, Lakshmi e Jayakrishnan, em 2002, realizaram um estudo, no qual sintetizaram microesferas ocas de policarbonato (PC) carregadas de piroxicam, capazes de flutuar em fluidos gástricos e intestinais artificiais, através da técnica de evaporação do solvente. A liberação in vitro de piroxicam num fluido gástrico artificial a $37^{\circ} \mathrm{C}$ mostrou um efeito de liberação rápida não significativo e que a quantidade de fármaco liberada com o tempo aumentou até às 8 horas, tempo a partir do qual não se liberaram quantidades significativas de fármaco. No fluido intestinal a liberação foi mais rápida e contínua e, para maiores cargas de fármaco, liberação acumulativa alcançou 90\% em 8 horas. A análise farmacocinética mostrou que a biodisponibilidade do piroxicam das microesferas de PC era 1,4 vezes superior em relação ao fármaco livre e 4,8 vezes superior em relação a adição de microesferas e dose de carregamento individualmente. $\mathrm{O}$ estudo demonstrou que as microesferas de PC permitem liberação sustentada de piroxicam por período de tempo mais longo e um aumento da sua biodisponibilidade.

El-Gibaly, em 2002, sintetizou microesferas ocas contendo melatonina por interação iônica de quitosana e um tensioativo carregado negativamente, o dioctil sulfosuccinato de sódio (DOS). Os perfis de liberação revelaram uma taxa de cedência de fármaco constante quando em fluido gástrico artificial, sendo a liberação do fármaco retardada por várias horas $(1,7 \mathrm{~h}-6,7 \mathrm{~h})$, dependendo de fatores de processamento, enquanto que a liberação a partir de microesferas não-flutuantes (quitosana/ tripolifosfato) era quase imediata. A maior parte das 
microesferas desenvolvidas mostraram períodos de flutuação superiores a 12 horas em biofluidos artificiais. Estudos de dilatação mostraram que as microesferas de DOS/quitosana inchavam menos e não se dissolviam em fluido gástrico artificial por pelo menos 3 dias, enquanto que microesferas de quitosana/tripolifosfato inchavam claramente e perdiam marcadamente a sua integridade ao fim de 5 horas.

Sato et al., em 2004, desenvolveram microesferas esféricas contendo uma cavidade esférica revestida por uma camada de polímero dura, que mostraram excelente flutuabilidade no estômago. As microesferas foram preparadas usando o método da difusão de um solvente emulsionado usando polímeros acrílicos dissolvidos em mistura de diclorometano e etanol. As microesferas contendo riboflavina foram administradas a três voluntários saudáveis e o nível de riboflavina absorvida, controlado através da excreção na urina. A meia-vida de excreção após a administração de microesferas com tamanho de partícula entre 500-1000 $\mu$ exibindo elevada capacidade de flutuar, era maior do que o das microesferas com tamanho de partícula menor que $500 \mu$ e baixa capacidade de flutuação. As microesferas preparadas a partir da mistura de hidroxipropilmetilcelulose em diferentes proporções com riboflavina mostraram melhoria nas propriedades de liberação da riboflavina. Assim, a excreção urinária total de riboflavina era, em grande parte, influenciada pela sua liberação, que dependia da proporção de HMPC. Assim, as microesferas ideais são aquelas com elevada capacidade de flutuação e capacidade de liberação de fármaco suficiente.

Umamahesshwari et al., em 2003, desenvolveram microsferas de policarbonato (PC) como transportadores de fármacos para combater infecções por Helicobacter pylori. Com o objectivo de aumentar o efeito antiHelicobacter pylori do ácido acetoidroxâmico (AHA) foram preparadas microsferas de PC pelo método de evaporação do solvente. Os estudos in vitro confirmaram as excelentes características de flutuabilidade das microsferas de PC. Estudos in vivo demonstraram que a quantidade de AHA necessária para se observar um efeito antiHelicobacter pylori era 10 vezes inferior no caso da utilização de microsferas de $\mathrm{PC}$ em relação à administração isolada do fármaco, devido ao maior tempo de permanência gástrica proporcionado pelas microsferas de PC.

Sriamornsak, Thirawong e Puttipatkhachorn, em 2004, desenvolveram um novo método de preparação de pastilhas de gel de pectinato de cálcio com óleo incorporado capazes de flutuar no conteúdo gástrico. As pastilhas foram preparadas pelo método de emulsificação-gelificação. As pastilhas flutuavam quando se usava uma quantidade suficiente de óleo. O tipo e a percentagem de óleo usado nas pastilhas era determinante para as suas características de flutuação.

De la Torre, Torrado e Torrado, em 2005, desenvolveram um sistema de liberação modificada com o objetivo de aumentar a eficácia da amoxicilina no tratamento de infecções de Helicobacter pylori. Para tal utilizaram um complexo poliônico de ácido poliacrílico, quitosana e amoxicilina com o objetivo de obter um hidrogel que aumentasse o volume, o que lhe permitia flutuar de modo a prolongar o GRT. O complexo poliônico (PAA:CS:A 2.5:5:2) mostrou liberação sustentada em suco gástrico artificial (sem enzimas) a pH 4,0. Este tipo de complexo protege a amoxicilina da degradação hidrolítica no suco gástrico. Um estudo in vivo realizado em voluntários saudáveis usando o teste de respiração de $\left[\mathrm{C}^{13}\right]$-ácido octanoico demonstrou que o hidrogel prolongava o GRT em 3 horas.

Losi, Bettini, Colombo, Peppas et al., em 2006, desenvolveram um sistemas que consiste em dois comprimidos na forma de abóboda unidos por ultrassons, formando, assim, um núcleo vazio, que permite a flutuação do sistema farmacêutico desde o instante inicial. A liberação do fármaco é conseguida pelo uso de polímeros hidrofílicos como o HPMC.

\section{CONCLUSÃO}

Os dispositivos de liberação modificada podem ser classificados genericamente em dispositivos efervescentes e não-efervescentes mediante o processo de liberação. Este tipo de dispositivos apresenta-se como uma aproximação racional à necessidade de modular a liberação de fármacos ao nível do trato gastrointestinal. Estes dispositivos apresentam vantagens sobretudo no caso de fármacos que são principalmente absorvidos no estômago ou parte proximal do intestino delgado. Apresentam, no entanto, alguns inconvenientes, tais como a difícil quantificação da eficácia de flutuação no estado de jejum ou no estado alimentado, as grandes diferenças fisiológicas evidenciadas entre homens e mulheres ao nível de esvaziamento gástrico, as possíveis influências de inúmeros fatores fisiológicos como a ansiedade e a difícil correlação entre flutuação in vitro e aumento da permanência gástrica.

Os materiais poliméricos apresentam-se atualmente como a maior fonte de matéria-prima para o desenvolvimento de sistemas flutuantes, visto que a maioria possibilita, num único material, a junção de duas características essenciais nestes tipos de dispositivos: capacidade de flutuar e de liberar de forma controlada o fármaco. 


\section{ABSTRACT}

\section{Pharmaceutical gastro-retentive systems}

Gastro-retentive systems are often produced in order to modulate drugs release from pharmaceutical forms and in this way to increase drug residence time in the gastrointestinal tract. One of the most interesting strategies consists in the preparation of floating devices. These can be classified into two groups: effervescent systems and non-effervescent systems. A review of what has been done in the last years is presented in this article.

UNITERMS: Gastro-retentive systems. Floating devices. Effervescent systems. Modified release pharmaceutics.

\section{REFERÊNCIAS BIBLIOGRÁFICAS}

AMINABHAVI, M.; ANANDRAO, R.; KULKARNI, M.D.; KUMARESH, S.; SOPPIMATH T. Development of hollow microspheres as floating controlled release systems for cardiovascular drugs: preparation and release characterisitics. Drug Dev. Ind. Pharm., v.27, n.10, p.1107-1114, 2001.

ATYABI F., SHARMA H. L.,MOHAMMAD H. A. H, FELL J.T. A novel floating system using ion exchange resins. Proc. Int. Symp. ControI. Release Bioact. Mater., v.21, p.806-807, 1994.

ATYABI F., SHARMA H. L.; MOHAMMAD H. A. H, FELL J.T. In vivo evaluation of a novel gastric retentive formulation based on ion exchange resins. J. Control. Release, v.42, p. 105-113, 1996.

BAGGESEN, H.; BECHGAARD, S. Propoxyphene and norpropoxyphene: influence of type of controlled release formulation on intra-and inter-subject variations. $J$. Pharm.Sci., v.69, n.11, p.1327-1330, 1980.

BAUMGARTNER, S.; KRISTL, J.; VRECER, F.; VODOPIVEC, P.; ZORKO,B. Optimisation of floating matrix tablets and evaluation of their gastric residence time. Int. J. Pharm., v.195, n.1-2, p.125-135, 2000.

BERNABEI, M.T.; IANNUCCELLI, V.; COPPI, G.; CAMERONI, R. Air compartment multiple-unit system for prolonged gastric residence. Part I. Formulation study. Int. J. Pharm., v.174, p. 47-54, 1998.
BI, D.; WEI, Z.; ZHANFENG, Y. Design and evaluation of a two-layer floating tablet for gastric retention using cisapride as a Model Drug. Drug Dev. Ind. Pharm, v.27, n.5, p.469-474, 2001.

BOLTON, S.; DESAI, S. A floating controlled-release drug delivery systems: in vitro-in vivo evaluation. Pharm. Res., v.10, p.1321-1325, 1993.

BOLTON, S.; DESAI, S. Floating sustained release therapeutic compositions. US Patent 4,814, 179, March $21,1989$.

BURI, P.; ROUGE, N.; DOELKER, E. Drug absortion sites in gastrointestinal tract and dosage forms for site- specific delivery. Int. J. Pharm., v.136, p.117-139, 1996.

CHANG, H. S. H.; PARK, P.; KELLY, ROBINSON, J. R. Bioadhesive polymers as platforms for oral controlled drug delivery II. Synthesis and evaluation of some swelling, water insoluble polymers. J. Pharm. Sci., v.74, n.4, p.399-405, 1985

CHITNIS, V.S.; MALSHE, V.S.; LALLA, I.K. Bioadhesive polymers-synthesis, evaluation and application in controlled release tablets. Drug Dev. Ind. Pharm., v.17, p.879-892, 1991.

COLLETT, L.; WHITEHEAD, J. R.; FELL, J.T. Development of a gastroretentive dosage form. Eur. I Pharm. Sci., v.4, (suppl.), S182, 1996.

COLLETT, L.; WHITEHEAD, J.R.; FELL, J. T.; SHARMA, H.L.; SMITH, A-M. Floating dosage forms: an in vivo study demonstrating prolonged gastric retention. J. Control. Release, v.55, p.3-12, 1998.

COLLET, L.; FELL, J.T..; WHITEHEAD, J.R. Prolonged gastric retention using floating dosage forms. Pharm. Technol., v.24, n.3, p.82-90, 2000.

COPPI, G.; IANNUCCELLI, V. R.; SANSONE, G. FROLLA. Air compartment multiple-unit system for prolonged gastric residence. Part II In vivo evaluation. Int. J. Pharm., v.174, p.55-62, 1998.

DAVE, S.B.; AMIN, A.F.; PATEL, M. Gastoretentive drug delivery system of ranitidine hydrochloride: formulation and in vitro evaluation. AAPS Pharmscitech, v.5, n.2, p.34, 2004. 
DAVIS, S.S.; STOCKWELL, A.F.; WALKER, S.E. In vitro evaluation of alginate gel systems as sustained release drug delivery systems. J. Control. Release, v.3, n.167-175, 1986.

DEASY, P.B.; HILTON, A.K. In vitro and in vivo evaluation of an oral sustained-release floating dosage form of amoxycillin trihydrate. Int. J. Pharm., v.86, p.79-88,1992.

DENNIS, A.; TIMMINS, P.; LEE, K., Buoyant controlled release powder formulation. US Patent 5, 169,638, December 8, 1992.

DESHPANDE, A. A.; RHODES, C. T.; SHAH, N. H.; MALICK, A. W. Controlled-release drug delivery systems for prolonged gastric residence: an overview. Drug. Dev. Ind. Pharm., v.22, n.6, p.531-539, 1996.

ESHRAGHI, J.; YANG, L.; FASSIHI, R. A new intragastric delivery system for the treatment of 1 Helicobacter pylori associated gastric ulcer: in vitro evaluation. J. Control. Release, v.57, p.215-222, 1999.

FELL, J. T. Delivery systems for targeting to specific sites in gastrointestinal tract. J. Pharm. Pharmacol., v.51, (suppl.), p.41, 1999.

FRANZ, M.R.; OTH, M.P. Sustained release, bilayer buoyant dosage form. US Patent 5, 232, 704, August 3, 1993.

FRIEND, D.R.; RUBINSTEIN, A. Polymeric sitepharmacotherapy. Chichester: Wiley, 1994.

FRYERS, G.R.; TODD. R.S. Cholestyramine compositions and method for treating biliary gastritis. US Patent 4, 172, 120, 1979.

HARRIGAN, R.M. Drug delivery device for preventing contact of undissolved drug with the stomach lining. US Patent 4,055, 178, 1977.

HINO, T.; KAWASHIMA, Y.; NIWA, H.; TAKEUCHI, T.; ITO, Y., Preparation of multiple hollow microspheres (microballoons) with acrylic resin containing tranilast and their drug release characteristic (in vitro) and floating behaviour (in vivo). J. Control. Release, v.16, p.279-290, 1991.

HINO, T.; KAWASHIMA, Y.; NIWA, H.; TAKEUCHI, T.; ITO, Y.; Hollow microspheres for use as a floating controlled drug delivery system in the stomach. J. Pharm. Sci., v.81, p.135-140, 1992.
IBRAHIMEL-GIBALY. Development and in vitro evaluation of novel floating quitosano microcapsules for oral use: comparison with non-floating chitosan microspheres. Int. J. Phar., v.249, p.7-21, 2002.

ICHIKAWA, M.; WATANABE, S.; MIYAKE, Y. Granule remaining in stomach. US Patent 4, 844,905, 1989.

INOUYE, K.; MACHIDA, Y.; SANNAN, T.; NAGAI, T. Buoyant sustained release tablets based on chitosan. Drug Des. Del., v.2, p.165-175,1988.

INOUYE, K.; MACHIDA, Y.; SANNAN, T.; NAGAI, T. Buoyant sustained release granules based on chitosan. Drug Des. Del., v.4, p.55-67,1989.

ISHINO, Y.; WATANABE, S.; KAYANO, M.; MIYAO, K. Solid therapeutic preparation remaining in stomach. US Patent 3,976, 764,1976.

JAYAKRISHNAN, A.; THANOO, B.C.; SUNNY, M.C. Oral sustained-release drug delivery systems using polycarbonate microspheres capable of floating on the gastricfluid. J. Pharm. Pharmacol., v.45, p.21-24, 1993.

JAYAKRISHNAN, A.; JOSEPH, N.J.; LAKSHMI, S. A floating-type oral dosage form for piroxicam based on hollow polycarbonate microspheres: in vitro and in vivo evaluation in rabbits. J. Control. Release, v.79, p.71-79, 2002.

KAWASHIMA,Y.; SATO, Y.; TAKEUCHI, T.; YAMAMOTO, $\mathrm{H}$. In vitro and in vivo evaluation of riboflavin-containing microballoons for a floating controlled drug delivery system in healthy humans. Int. J. Phar., v.275, p.97-107, 2004.

LOSI, E.; BETTINI, R.; SANTI, P.; SONVICO, F.; COLOMBO, G.; LOFTHUS, K.; COLOMBO, P.; PEPPAS, N.A. Assemblage of novel release modules for the development of adaptable drug delivery systems. $J$. Control. Release, v.111, n.1-2, 10, p.212-218, 2006.

MATHARU, R. S.; SANGHAVI, N. M. Novel drug delivery system for captopril. Drug Dev. Ind. Pharm., v.18, p.1567-1574, 1992.

MICHAELS, A.S. Drug delivery device with self actuated mechanism for retaining device in selected area. US Patent 3, 786, 813, 1974. 
MITRA, S.B. Sustained release oral medicinal delivery device. US Patent 4, 451,260, 1984.

MIYAZAKI, S.; YAMAGUCHI, H.; YOKOUCHI, C.; TAKADA, M.; HOU, W.M. Sustained-release and intragastric-floating granules of indomethacin using chitosan in rabbits. Chem. Pharm. Bull., v.36, p.40334038, 1988.

NAKAMIEHI, K.; USHIMARU, K.; SAITO, R. Pharmaceutical preparations and a method of manufacturing them. US Patent 4,702,918, 1987

PARK, K.; ROBINSON, J. Bioadhesive polymers as platform for oral-controlled drug delivery: method to study bioadhesion. Int. J. Pharm., v.19, n.1, p.107-127, 1984.

RITSEHEL, W.A. Targeting in the gastrointestinal tract: new approaches; Methods Find. Exp. Clin. Pharmacol., v.13, p.313-336, 1991 .

SHETH, P.R.; TOSSOUNIAN, J.L. Sustained release pharmaceutical capsules. US Patent 4, 126,672, 1978.

SHETH, P. R.; TOSSOUNIAN J.L. Novel sustained release tablet formulations. US Patent 4, 167,558, 1979.

SHETH P.R.; TOSSOUNIAN J.L. Sustained release tablet formulations. US Patent 4, 140, 755, 1979.
SHETH, P.R.; TOSSOUNIAN J.L. The hydrodynamically balanced system (HBSTM): a novel drug delivery system for oral use. Drug Dev. Ind. Pharm., v.10, p.313-339, 1984.

TORRADO, S.; DE LA TORRE, P.M.; TORRADO, G. Poly (acrylic acid) chitosan interpolymer complexes for stomach controlled antibiotic delivery. J Biomed Mater Res B Appl Biomater, v.72, n.1, p.191-197, 2005.

UMAMAHESHWARI, R. B.; JAIN, N. K.; JAIN, S. New approach in gastroretentive drug delivery system using cholestyramine. Drug Del., v.10, p.151-160, 2003.

UMEZAWA, H. Pepstatin floating minicapsules. US Patent 4, 101,650,1978

XIAOQIANG, X.; MINJIE, S.; FENG, Z.; YIQIAO, H. Floating matrix dosage form for phenoporlamine hydrochloride based on gas forming agent: In vitro and in vivo evaluation in healthy volunteers. Int. J.Pharm., v.310, n.1-2, p.139-145, 2006.

Recebido para publicação em 04 de maio de 2005. Aceito para publicação em 26 de junho de 2006 